\title{
Investigation of extended blinks and interblink intervals in subjects with and without dry eye
}

This article was published in the following Dove Press journal:

Clinical Ophthalmology

13 February 2013

Number of times this article has been viewed

\author{
John D Rodriguez' \\ George W Ousler III' \\ Patrick R Johnston' \\ Keith Lane' \\ Mark B Abelson ${ }^{1,2}$ \\ 'Ora, Inc, Andover, MA, ${ }^{2}$ Schepens \\ Eye Research Institute and Harvard \\ Medical School, Boston, MA, USA
}

Correspondence: John Rodriguez

ORA, Inc, 300 Brickstone Square

Andover, MA 01810, USA

Tel +l 9786858900

Fax + I 9786890020

Email jrodriguez@oraclinical.com
Background: The purpose of this study was to investigate the occurrence and duration of extended blinks $\geq 70 \mathrm{msec}$ and their associated interblink intervals in normal subjects and in subjects with mild to moderate dry eye.

Methods: This single-center, prospective, double-blind study included 11 subjects with dry eye and eight subjects with normal eyes. Extended blinks were defined as lid closure in at least two successive video frames ( $\geq 70 \mathrm{msec}$ ). Digital video imaging of each subject's eyes was recorded while the subject viewed a 10-minute documentary. The subjects did not know that blink was the outcome being measured. Following capture, the videos were manually analyzed in a masked fashion for the occurrence of extended blinks. The length of the interblink interval (ie, time between blinks) before and after these extended blinks (the interblink interval ratio) was calculated, as well as differences in lid contact times.

Results: The dry eye group had a median extended blink duration which was 2.53 times longer than that of the normal group. For subjects with dry eye, interblink intervals post-extended blink were significantly longer than interblink intervals pre-extended blink $(P<0.001)$. Interblink intervals did not lengthen significantly in normal subjects. In both groups, the duration of the extended blink was significantly $(P=0.001)$ and positively correlated with interblink interval ratio (post-extended to pre-extended blink interblink interval), such that for each doubling of extended blink duration, the interblink interval ratio increased by $10 \%$. Blinks longer than one second in duration occurred almost exclusively in subjects with dry eye.

Conclusion: This study reports three central findings: blink duration tended to be longer in subjects with dry eye; a lengthening of the interblink interval after an extended blink occurred in subjects with dry eye but not in those without dry eye; and a longer blink duration was associated with a significantly increased interblink interval ratio in all subjects.

Keywords: dry eye, interblink intervals, visual function, visual tasks, diagnostic model

\section{Introduction}

Dry eye has been formally defined as "a multifactorial disease of the tears and ocular surface that results in symptoms of discomfort, visual disturbance, and tear film instability, with potential damage to the ocular surface". ${ }^{1}$ The pathogenic mechanisms of dry eye may result in blink modification. Blinks have been classified by their specific kinematic properties, eg, by duration of lid movement to full closure, time of closure when vision is occluded, and time to reopening, as well as by their associated neural activity or other endogenous or exogenous stimuli. ${ }^{2}$ Distinctive blink phenomena described by various investigators include reflex, endogenous, conditioned and voluntary responses, nonblink closures, and microsleeps. ${ }^{2-9}$ The relevant time scale for eyelid movements described as blinks is generally held to 
be between $100 \mathrm{msec}$ and $400 \mathrm{msec}^{2}$ Proper resolution of reflex blink motions requires frame rates of several hundred frames per second or electro-oculogram/electromyogram signal traces.

Our specific parameters of interest are lid contact times and lid closures. During a typical full blink, lid contact time can vary from less than $10 \mathrm{msec}$ to $80 \mathrm{msec}^{2}$ In the present work, we observed lid closures of up to several seconds that we refer to as "extended blinks". We concentrated on lid closures lasting at least $70 \mathrm{msec}$, recognizing that the longer the lids remain closed, the further away the event is from a conventional blink. These events have been given a variety of names, depending on the related psychophysical state or visual task, including nonblink closures, ${ }^{2}$ behavioral microsleeps or "lapses", ${ }^{4}$ or microsleeps. ${ }^{5}$ Eyelid closures of prolonged duration may result from various factors, and are usually studied in the context of alertness, attention, and fatigue. ${ }^{4-9}$ In young normal subjects who are not sleep-deprived, blink-associated lapses during an extended visual task have been observed to occur with frequency. ${ }^{4}$ In fatigue research, long lid closures, accompanied by sleep-like electroencephalographic activity, are defined as "microsleeps". $5-9$

Knowing that dry eye is associated with increased blink rates and shorter interblink intervals, ${ }^{10,11}$ we hypothesized that ocular surface stress might also result in an increase in anomalous phenomena, such as microsleeps and nonblink closures or, as we describe, extended blinks. Certainly, so-called "squeeze blinks", which are long-duration lid closures accompanied by elevated pressure on the lid margin, have been shown to increase lipid expression ${ }^{12}$ and tear film thickness. $^{13}$

The primary goal of blinking is protection of the ocular surface and restabilization of the transparent, homogeneous tear film necessary for uninterrupted vision. ${ }^{14}$ The observed findings in dry eye of increased blink rates, decreased blink intervals, and changing patterns of blinks are thought to add significantly to the constellation of signs and symptoms leading to compromised visual function. ${ }^{10-13,15-18}$

We have previously identified patterns of partial, full, twitch, and squeeze blinks, and the association of incomplete blinks with superficial punctate keratitis. ${ }^{16}$ Blinking is an important factor in the study of dry eye, and recognition of variations in blink patterns is critical to understanding and categorizing subgroups that might respond differently to treatment.

In the present study, we analyzed extended lid contact times (ie, extended blinks) of $70 \mathrm{msec}$ and longer in duration.
The objective was to define the occurrence and duration of such blinks in normal and dry eye subjects, and the nature of the interblink interval before and after an extended blink.

\section{Materials and methods Study design and subjects}

This single-center study was conducted in 11 subjects with dry eye (nine women, two men, mean age 61.8 years) and eight normal subjects (six women, two men, mean age 26.8 years). The study was performed according to a protocol approved by an external independent review board (Alpha IRB, San Clemente, CA, USA) and written informed consent was obtained prior to initiation of any study procedures. Subjects were at least 18 years of age and were required to have a best corrected visual acuity of +0.6 logMAR (logarithm of the maximum angle of resolution) or better in each eye based on the Early Treatment of Diabetic Retinopathy Study chart. Subjects were required to avoid ophthalmic medications, including artificial tear substitutes, for 2 hours prior to the study visit. Any systemic medications suspected to cause ocular drying must have been taken on a stable dosage regimen for at least 14 days prior to the study visit.

Subjects were excluded from the study if they wore contact lenses, had any ocular inflammation or infection, preauricular lymphadenopathy, or any significant illness that the investigator felt could have put the subject at risk or interfered with the study findings.

Subjects with dry eye and normal subjects were selected based on a reported/lack of history of dry eye, respectively, and the status of each subject was confirmed by fluorescein staining conducted after video capture. Fluorescein staining was assessed by the investigator using a slit lamp following instillation of $5 \mu \mathrm{L}$ of $2 \%$ preservative-free sodium fluorescein into the inferior conjunctival cul de sac, as previously described. ${ }^{19}$ Staining was graded in precisely defined regions of the ocular surface using a 0-4-point scale ( 0 , none; 4 , worst staining). Subjects with staining $\geq 1.5$ in one or more of the five regions were confirmed to have dry eye; subjects with staining $<1$ in all of the five regions considered were confirmed as having normal eyes.

\section{Video monitoring environment}

Each subject was instructed to view a 10-minute documentary in an isolated room under standardized lighting, temperature, and humidity conditions. Because awareness can substantially alter blink rates, subjects were only told that different aspects of the tear film were being studied and not specifically blinking. 


\section{Blink measurement}

Blink information was obtained from digital video imaging captured with a digital micro camera (Imaging Development Systems, Obersulm, Germany) while subjects watched a 10-minute documentary. The camera was mounted to a headset and directed towards the eye so that eyelid movement could be captured noninvasively. An extended blink was defined as lid closure (ie, lid contact time) in at least two successive video image frames of $35 \mathrm{msec}$. Once extended blinks were identified, the single interblink interval (IBI) immediately preceding and immediately following each extended blink was identified and measured. The length of the IBI immediately following the extended blink compared with the length of the IBI immediately preceding the extended blink (the IBI ratio) was calculated as the outcome of interest (Figure 1). The differences in lid contact time for extended blinks were compared between the groups.

\section{Statistical analysis}

Group means for the log of the post/pre IBI ratio were estimated using a correlated normal linear model based on estimating equation methods. Robust standard errors were obtained assuming a working exchangeable correlation structure. Under an assumption of log-normality, group medians and their ratio were obtained on the original scale upon exponentiation. The same method was used to estimate group medians and their ratio for extended blink duration.
The relationship between the post/pre IBI ratio and extended blink duration was obtained by a correlated linear regression of the log of the post/pre IBI ratio against the log of the extended blink duration. This log-log model implies a power relationship between the post/pre IBI ratio and extended blink duration on the original scale. All models were fit using the GENMOD procedure of SAS version 9.2 (SAS Institute Inc, Cary, NC, USA). The null hypothesis tested was that a given IBI ratio was 1 (ie, pre-IBIs and post-IBIs were not different).

\section{Results}

Eight of the 11 subjects with dry eye (72.7\%), and seven of the eight normal subjects had observable extended blinks $(87.5 \%)$. Of the subjects with dry eye, seven were female, one was male, and the mean age was 60 years. The clinical characteristics of dry eye in these eight subjects are shown in Table 1. The normal subjects consisted of six females and one male, with a mean age of 27.3 years. The dry eye group had a total of 68 extended blinks and the normal group had a total of 52 extended blinks, cumulatively, over the course of the 10-minute viewing period. Median durations of extended blinks were calculated for each subject. The range of these medians in the dry eye group was $0.13-3.93$ and in the normal group was $0.07-1.33$. Extended blink number per subject was similar between the groups, with subjects in the dry eye group having 1-24 extended blinks (median 5.5) and subjects in the normal group having 1-23 extended blinks (median 2).

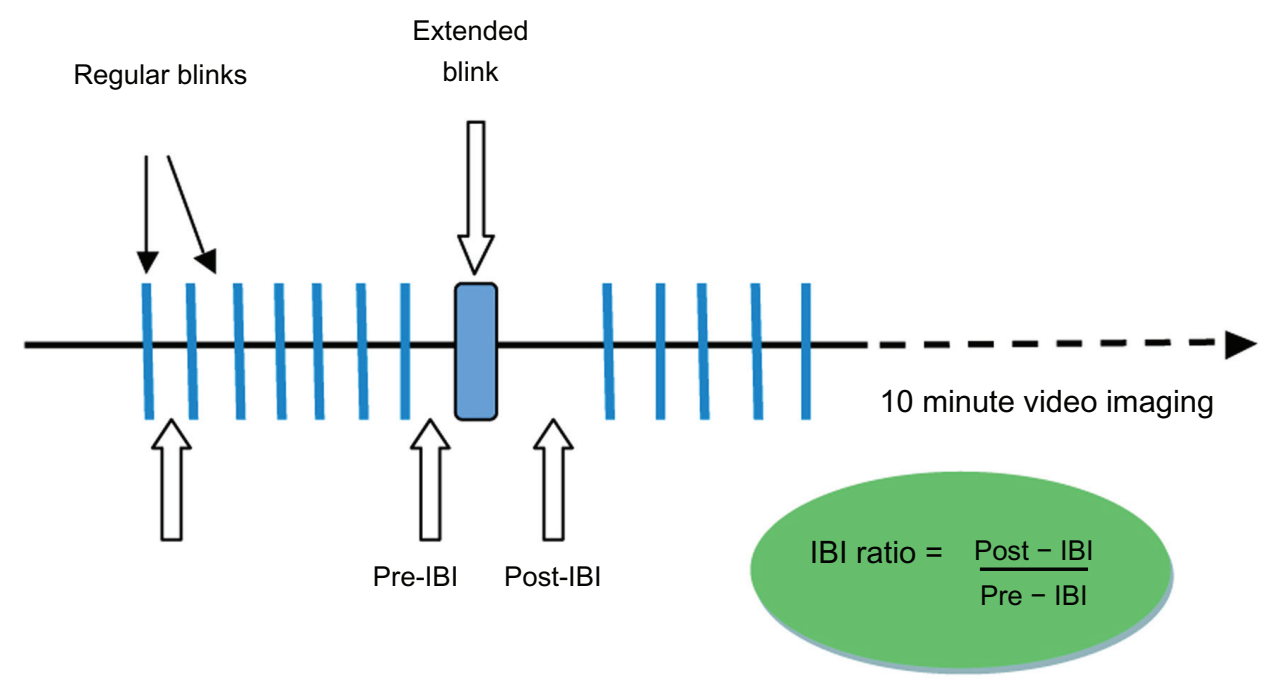

Figure I Extended blink patterns.

Notes: Schematic representation of the time sequence investigated in this study: interblink intervals before and after extended blinks, which were defined as events occurring when a subject's lids were closed for two successive video frames of $35 \mathrm{msec}$ in duration, ie, $70 \mathrm{msec}$.

Abbreviation: IBI, interblink interval. 
Table I Characteristics of the eight subjects with dry eye and extended blinks

\begin{tabular}{|c|c|c|}
\hline Variable & Mean (SE) & Range \\
\hline Age (years) & $60 \pm 5.02$ & $37-73$ \\
\hline Visual acuity $O D$ & $0.058 \pm 0.03$ & $-0.02-0.02$ \\
\hline Visual acuity OS & $0.003 \pm 0.01$ & $-0.06-0.04$ \\
\hline TFBUT I OD (seconds) & $5.15 \pm 1.51$ & $2.06-13.19$ \\
\hline TFBUT I OS (seconds) & $4.22 \pm 0.522$ & $2.5-5.69$ \\
\hline TFBUT 2 OD (seconds) & $4.74 \pm 0.995$ & $2.21-8.62$ \\
\hline TFBUT 2 OS (seconds) & $3.99 \pm 0.750$ & $2.19-7.78$ \\
\hline Discomfort OD (0-4 scale) & $1.00 \pm 0.267$ & $0-2$ \\
\hline Discomfort OS (0-4 scale) & $0.75 \pm 0.250$ & $0-2$ \\
\hline Burning $O U(0-4$ scale $)$ & $1.38 \pm 0.532$ & $0-3$ \\
\hline Dryness OU (0-4 scale) & $1.63 \pm 0.420$ & $0-3$ \\
\hline Grittiness OU ( $0-4$ scale) & $1.5 \pm 0.378$ & $0-3$ \\
\hline Stinging $O U$ ( $0-4$ scale) & $1.63 \pm 0.532$ & $0-4$ \\
\hline Inferior corneal staining OD (0-4 scale) & $2.57 \pm 0.277$ & I.5-3.5 \\
\hline Inferior corneal staining OS (0-4 scale) & $2.50 \pm 0.345$ & $1-3.5$ \\
\hline Superior corneal staining OD ( $0-4$ scale) & $2.36 \pm 0.180$ & $2-3$ \\
\hline Superior corneal staining OS ( $0-4$ scale) & $2.43 \pm 0.223$ & $1.5-3$ \\
\hline Central corneal staining OD (0-4 scale) & $1.93 \pm 0.442$ & $0-3$ \\
\hline Central corneal staining OS ( $0-4$ scale $)$ & $1.86 \pm 0.460$ & $0-3$ \\
\hline $\begin{array}{l}\text { Temporal conjunctival staining OD } \\
(0-4 \text { scale) }\end{array}$ & $2.071 \pm 0.277$ & $1-3$ \\
\hline $\begin{array}{l}\text { Temporal conjunctival staining OS } \\
\text { ( } 0-4 \text { scale) }\end{array}$ & $2.00 \pm 0.309$ & $1-3$ \\
\hline Nasal conjunctival staining OD ( $0-4$ scale) & $2.00 \pm 0.309$ & $1-3$ \\
\hline Nasal conjunctival staining OS (0-4 scale) & $1.93 \pm 0.442$ & $0-3.5$ \\
\hline
\end{tabular}

Demographic, blink duration, and IBI data are summarized by group in Table 2 .

Model-based estimates of group medians for extended blink durations are presented in Table 3. The dry eye and normal eye groups had median extended blink durations of

Table 2 Sample means and medians of extended blinks identified in a subgroup of eight of II subjects with dry eye and seven of eight normal subjects

\begin{tabular}{lll}
\hline Variable & Normal subjects & Dry eye subjects \\
\hline $\begin{array}{l}\text { Subjects with extended blinks } \\
\text { (n) }\end{array}$ & $7 / 8$ total & $8 / 1$ I total \\
Mean age (years) & $(87.5 \%)$ & $(72.7 \%)$ \\
Gender & 27.3 years & 57.5 years \\
& 6 females, & 7 females, \\
I male & I male \\
Extended blinks (total, $\mathrm{n})$ & 52 & 68 \\
$\begin{array}{l}\text { Extended blinks (median, } \mathrm{n}) \\
\text { Duration of extended blink } \\
\text { (median, msec) }\end{array}$ & 2 & 5.5 \\
IBI pre-extended blink & 230 & 470 \\
(median, sec) & 3.13 & 1.93 \\
$\begin{array}{l}\text { IBI post-extended blink } \\
\text { (median, sec) }\end{array}$ & 3.43 & 2.90 \\
\hline
\end{tabular}

Abbreviation: IBI, interblink interval.
Table 3 Estimates from the correlated normal model: median extended blink duration in seconds in eight subjects with dry eye and seven normal subjects

\begin{tabular}{|c|c|c|c|c|}
\hline \multirow[t]{2}{*}{ Group } & \multirow{2}{*}{$\begin{array}{l}\text { Median* extended } \\
\text { blink duration } \\
\text { (seconds) }\end{array}$} & \multicolumn{2}{|c|}{$95 \% \mathrm{Cl}$} & \multirow[t]{2}{*}{$P$ value } \\
\hline & & Low & High & \\
\hline Dry eye $(n=8)$ & 0.59 & 0.24 & 1.46 & \\
\hline Normal $(n=7)$ & 0.23 & 0.11 & 0.49 & \\
\hline Dry versus normal eyes & 2.53 & 0.80 & 8.03 & 0.116 \\
\hline
\end{tabular}

Note: *Medians based on estimated correlated normal model.

Abbreviation: $\mathrm{Cl}$, confidence interval.

0.59 seconds and 0.23 seconds, respectively. Thus, the median duration was 2.53 times longer in dry eyes than in normal eyes (trend only, $P=0.116$ ).

The median pre-extended and post-extended blink IBIs were 1.93 seconds and 2.90 seconds, respectively, for subjects with dry eye and 3.13 seconds and 3.43 seconds, respectively, for normal subjects. This difference from post-IBI to pre-IBI was only significant in subjects with dry eye $(P<0.001)$. In normal subjects, IBI duration was not significantly different before and after an extended blink $(P=0.214)$. Comparison between the two groups approached statistical significance $(P=0.090)$. Table 4 summarizes the IBI results by group. Both groups showed a similar statistically significant $(P=0.001)$ positive relationship between IBI ratio (post-extended to pre-extended blink IBI) and extended blink duration, namely that for each doubling of extended blink duration, the IBI ratio increased by $10 \%$ (Figure 2).

From Figure 2, a further frequency distribution of extended blinks is apparent. When the cutoff for duration of extended blinks was increased to greater than one second of lid contact time (defined here as "rescue blinks"), 29 blinks were isolated, 26 of which were from three subjects with dry eye and three of which were from three normal subjects. Thus, while the

Table 4 Estimates from the correlated normal model: median IBI before and after extended blinks and the relative IBI ratios in eight subjects with dry eye and seven normal subjects

\begin{tabular}{lllll}
\hline Group & $\begin{array}{l}\text { Median* post- } \\
\text { extended to pre- }\end{array}$ & \multicolumn{2}{l}{$\mathbf{9 5 \% \mathbf { C l }}$} & P value \\
\cline { 3 - 4 } & extended blink IBI & Low & High nn & \\
\hline Dry eye $(\mathrm{n}=8)$ & 1.34 & 1.23 & 1.46 & $<0.00 \mathrm{I}$ \\
Normal $(\mathrm{n}=7)$ & $\mathrm{I} .12$ & 0.93 & 1.35 & 0.214 \\
$\begin{array}{l}\text { Dry eye versus } \\
\text { normals }\end{array}$ & 1.19 & 0.97 & 1.46 & 0.090 \\
\hline
\end{tabular}

Note: *Medians based on estimated correlated normal model. Abbreviations: $\mathrm{Cl}$, confidence interval; IBI, interblink interval. 


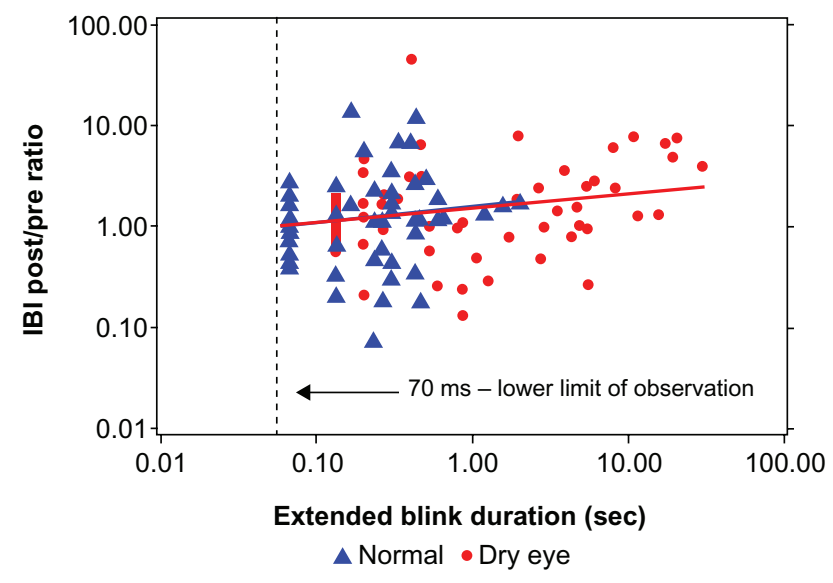

Figure 2 Post/pre IBI ratio versus extended blink duration.

Notes: Extended blink duration as a function of the ratio of IBI post-extended to pre-extended blink is shown for subjects with dry eye (red circles) and without dry eye (blue triangles). Both groups showed a similar statistically significant $(P=0.00 \mathrm{I})$ positive relationship, namely that for each doubling of extended blink duration, the IBI ratio increased by $10 \%$.

Abbreviation: IBI, interblink interval.

number of subjects demonstrating rescue blinks was the same, $89.7 \%$ of blinks one second or longer in duration occurred in subjects with dry eye, and only $10.3 \%$ in subjects with normal eyes. These data are summarized in Table 5.

\section{Discussion}

The central findings of this study indicate an alteration in blink patterns in dry eye subjects, whereby extended blinks tended to be longer in duration (although statistical significance was not met here due to the low sample size), IBIs were significantly longer after an extended blink, and blinks longer than one second occurred almost exclusively in subjects with dry eye. Furthermore, independent of dry eye status, as the duration of a blink increased, the ratio of post-extended to pre-extended blink IBI increased.

Table 5 "Extended blinks" versus "rescue blinks" (ie, more than one second of lid contact time) in the subgroups in which rescue blinks were identified, from the total population of eight subjects with dry eye and seven normal subjects

\begin{tabular}{lll}
\hline & $\begin{array}{l}\text { Extended blinks } \\
\geq \mathbf{7 0} \mathbf{~ m s e c}(\mathbf{n})\end{array}$ & $\begin{array}{l}\text { Rescue blinks } \\
\geq \text { I sec }(\mathbf{n})\end{array}$ \\
\hline $\begin{array}{l}\text { Normal subjects: } \mathbf{3 / 8} \\
\text { Subject number }\end{array}$ & \\
200 & 10 & $\mathrm{I}$ \\
201 & 2 & $\mathrm{I}$ \\
203 & $\mathrm{I}$ & $\mathrm{I}$ \\
Dry eye subjects: 3 /II & & \\
Subject number & & \\
I00 & 7 & 5 \\
I02 & 4 & 3 \\
III & 24 & 18 \\
\hline
\end{tabular}

We know that subjects with dry eye have shorter IBIs and increased blink rates and that these might affect visual function. ${ }^{10-13,16-18}$ Longer IBIs might result in fewer disturbances when performing visual tasks. Blink frequency is known to be a function of task, with high concentration tasks such as reading requiring a drastic lowering of blink frequency to maintain visual and cognitive processing, while much higher blink rates are observed during conversation and watching television. ${ }^{15,20-22}$ Blink frequencies and patterns as a function of task/visual processing/environmental conditions might be significantly altered when the stress of an inadequate tear film is an added variable.

Ocular stress and fatigue stress are parallel phenomena in the patient with dry eye. ${ }^{23}$ Eye fatigue has been shown to affect more than $70 \%$ of subjects with dry eye. ${ }^{24}$ Much of the current fatigue research has firmly established the correlation of fatigue with ocular signs such as blink duration and frequency. ${ }^{5-9}$ Increased blink rate occurs in the early stages of fatigue and extended blinks in the later stages. If a hierarchy of compensatory blink behavior can be determined in the subject with dry eye, considerable information about relative discomfort levels, hindrance of visual tasking, and the disease state may be obtained.

Tracking of natural blink patterns on video allows for accurate, noninvasive assessments that can easily be obtained, offering an advantage over more intensive and potentially inaccurate forced stare measures, such as fluorescein staining, mean breakup area, and tear film breakup time, or subjective measures such as symptomatic breakup time and potentially ambiguous ocular discomfort assessments. ${ }^{25,26}$ We chose documentary viewing as the visual task during our blink experiments because this gave greater repeatability compared with primary gaze, as well as more closely approximating a common everyday activity. This intermediate task requires minimal concentration, because too complex a task would have decreased blink rates, ${ }^{15,21,22}$ as well as sitting in silence, given that conversation greatly increases blink rates. ${ }^{20}$ Nevertheless, subjects with and without dry eye were studied under identical conditions.

It was apparent from our results that a different phenomenon was occurring at very extended lid contact times, and that these were closures stimulated by intrinsic and/or extrinsic stress, which cannot be considered normal reflex blinking. We have termed these long closures "rescue blinks" to differentiate them from other types of extended blinks, and future studies will focus on this phenomenon. The variability in blink patterns and frequencies within and across subjects indicates that this highly adjustable 
compensatory mechanism can be fine-tuned to changes in every external and internal situation. This study has shown that extended blinks are relatively common, yet the total number of extended blinks in subjects with dry eye was elevated compared with that in normal subjects, and rescue blinks of longer than one second occurred almost exclusively in subjects with dry eye. Furthermore, lengthening of the IBI after an extended blink was significant only in subjects with dry eye. These data suggest that extended blinks may serve more as a compensatory mechanism in subjects with dry eye relative to subjects without dry eye. It is not known whether the alterations in extended blink that we found were due to age-related changes in lids that might be a factor inherent to dry eye. Limitations of this study are its small sample size and the difference in age between subjects with and without dry eye. We plan in future studies to investigate additional factors within the dry eye population, including age, gender, and lid health, and their effect on blink patterns.

\section{Disclosure}

The authors report no conflicts of interest in this work.

\section{References}

1. [No authors listed]. The definition and classification of dry eye disease: report of the Definition and Classification Subcommittee of the International Dry Eye Workshop (2007). Ocul Surf. 2007;5:75-92.

2. Stern JA, Walrath LC, Goldstein R. The endogenous eyeblink. Psychophysiology. 1984;21:22-32.

3. Powers AS, Coburn-Litvak P, Evinger C. Conditioned eyelid movement is not a blink. J Neurophysiol. 2010;103:641-647.

4. Peiris MTR, Jones RD, Davidson PR, Carroll GJ, Bones PJ. Frequent lapses of responsiveness during an extended visuomotor tracking task in non-sleep-deprived subjects. J Sleep Res. 2006;15:291-300.

5. Schleicher R, Galley N, Briest S, Galley L. Blinks and saccades as indicators of fatigue in sleepiness warnings: looking tired? Ergonomics. 2008;51:982-1010.

6. Hakkanen H, Summala H, Partinen M, Tiihonen M, Silvo J. Blink duration as an indicator of driver sleepiness in professional bus drivers. Sleep. 1999;22:798-802.

7. Johns MW, Tucker A, Chapman R, Crowley K, Micheal N. Monitoring eye and eyelid movements by infrared refectance oculography to measure drowsiness in drivers. Somnologie (Berl). 2007;11:234-242.

8. Stern JA. Blink rate: a possible measure of fatigue. Hum Factors. 1994;36: 285-297.

Clinical Ophthalmology

\section{Publish your work in this journal}

Clinical Ophthalmology is an international, peer-reviewed journal covering all subspecialties within ophthalmology. Key topics include: Optometry; Visual science; Pharmacology and drug therapy in eye diseases; Basic Sciences; Primary and Secondary eye care; Patient Safety and Quality of Care Improvements. This journal is indexed on Submit your manuscript here: http://www.dovepress.com/clinical-ophthalmology-journal
9. Harrison Y, Horne JA. Occurrence of 'microsleeps' during daytime sleep onset in normal subjects. Electroencephalogr Clin Neurophysiol. 1996;98:411-416.

10. Tsubota K, Hata S, Okusawa Y, Egami F, Ohtsuki T, Nakamori K. Quantitative videographic analysis of blinking in normal subjects and patients with dry eye. Arch Ophthalmol. 1996;114:715-720.

11. Schlote T, Kadner G, Freudenthaler N. Marked reduction and distinct patterns of eye blinking in patients with moderately dry eyes during video display terminal use. Graefes Arch Clin Exp Ophthalmol. 2004;242: 306-312.

12. Korb DR, Baron DF, Herman JP, et al. Tear film lipid layer thickness as a function of blinking. Cornea. 1994;13:354-359.

13. Benedetto DA, Clinch TE, Laibson PR. In vivo observation of tear dynamics using fluorophotometry. Arch Ophthalmol. 1984;102: 410-412.

14. Holly FJ. Formation and rupture of the tear film. Exp Eye Res. 1973;15: 515-525.

15. Himebaugh NL, Begley CG, Bradley A, Wilkinson JA. Blinking and tear break-up during four visual tasks. Optom Vis Sci. 2009;86: E106-E114.

16. Abelson MB, Holly FJ. A tentative mechanism for inferior punctate keratopathy. Am J Ophthalmol. 1977;83:866-869.

17. White R, Rodriguez J, Lane KJ, Jonston P, Angjeli E, Abelson MB. Blink patterns in normal and dry eye subjects: beyond blink rate. Invest Ophthalmol Vis Sci. 2010;51:E-Abstract 3366.

18. Nally L, Ousler GW, Abelson MB. A correlation between blink rate and corneal sensitivity in a dry eye population. Invest Ophthalmol Vis Sci. 2003;44:E-Abstract 2488.

19. Abelson MB, Ousler GW 3rd, Nally LA, Wlech D, Krenzer K. Alternative reference values for tear film break up time in normal and dry eye populations. Adv Exp Med Biol. 2002;506(Pt B):1121-1125.

20. Bentivoglio AR, Bressman SB, Casetta E, Carretta D, Tonali P, Albanese A. Analysis of blink rate patterns in normal subjects. Mov Disord. 1997;12:1028-1034.

21. Cardona G, Garcia C, Seres C, Vilaseca M, Gispets J. Blink rate, blink amplitude, and tear film integrity during dynamic visual display terminal tasks. Curr Eye Res. 2011;36:190-197.

22. Freudenthaler N, Neuf H, Kadner G, Schlote T. Characteristics of spontaneous eyeblink activity during video display terminal use in healthy volunteers. Graefes Arch Clin Exp Ophthalmol. 2003;241: 914-920.

23. Walker PM, Lane KJ, Ousler GW, Abelson MB. Diurnal variation of visual function and the signs and symptoms of dry eye. Cornea. 2010;29: 607-612.

24. Toda I, Fujishima H, Tsubota K. Ocular fatigue is the major symptom of dry eye. Acta Ophthalmol. 1993;71:347-352.

25. Abelson R, Lane KJ, Angjeli E, Johnston P, Ousler G, Montgomery D. Measurement of ocular surface protection under natural blink conditions. Clin Ophthalmol. 2011;5:1349-1357.

26. Ousler GW, Gomes PJ, Welch D, Abelson MB. Methodologies for the study of ocular surface disease. Ocul Surf. 2005;3:143-154.

\section{Dovepress}

PubMed Central and CAS, and is the official journal of The Society of Clinical Ophthalmology (SCO). The manuscript management system is completely online and includes a very quick and fair peer-review system, which is all easy to use. Visit http://www.dovepress.com/ testimonials.php to read real quotes from published authors. 